\title{
Tien C. (T.C.) Tso, Recipient of the 2005 Tobacco Science Research Conference Lifetime Achievement Award
}

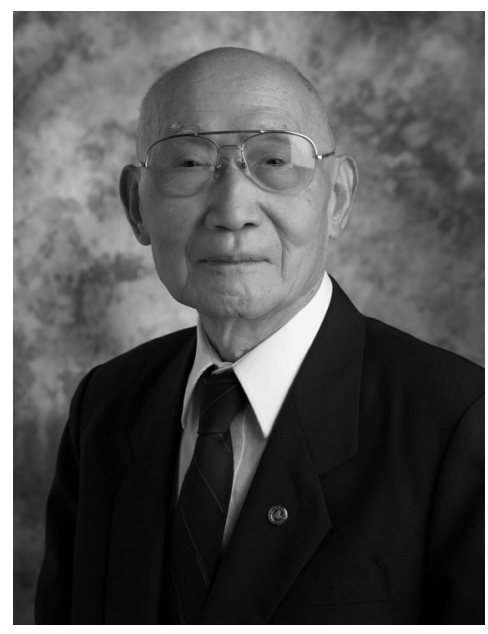

T.C., as he is known, is truly a Renaissance man. For over 30 years he was heavily involved in tobacco research with the United States Department of Agriculture (USDA) at the Beltsville Agricultural Research Center in Beltsville, Maryland. He maintained his relationship with tobacco research after this but continued to broaden his interests and contributions to agriculture, education and humanity from his base within USDA as Executive Director and Board Chairman of the Institute for International Development and Education in Agriculture and Life Sciences (IDEALS).

Dr. Tso received his B.S. degree from the University of Nanking, China in 1941 and his M.S. and Ph.D. from The Pennsylvania State University in 1944 and 1950 in agricultural biochemistry and agronomy. He was a post-doctoral scholar in the General Cigar Research Laboratories in 1950-51. In 1952 he joined the United States Department of Agriculture, Agricultural Research Service at Beltsville as a plant physiologist and has been with USDA to the present. It was from this base that he became the leader in the field of health effects related to tobacco use and was instrumental in getting many agencies and institutions working together on this issue, including other branches of USDA, National Cancer Institute, American Cancer Society and American Health Foundation.

Early on, T.C. published a series of papers on location and metabolism of tobacco alkaloids. He demonstrated that the rate of nicotine biosynthesis was greater in $N$. glutinosa than a converter $N$. tabacum and that nornicotine was detected in the roots earlier than in the shoot. In overmature $N$. glutinosa leaves nornicotine was formed in the absence of detectable nicotine. In $N$. tabacum converter plants nicotine biosynthesis was accelerated much more than demethylation of nicotine by topping. However, in $N$. glutinosa where the demethylation was active in immature plants the demethylation system was not limiting even when plants were topped and alkaloid content increased rapidly. Using ${ }^{15} \mathrm{~N}$ he demonstrated that tobacco alkaloid biosynthesis follows a stepwise series of reactions and possible alternate pathways. Nornicotine was formed in both the leaf and root and with the higher excess of ${ }^{15} \mathrm{~N}$ in nornicotine than nicotine suggested that it was formed independently in root or leaf of tobacco and not just in the leaf via demethylation. In further experiments using triple labels $-{ }^{14} \mathrm{C},{ }^{3} \mathrm{H}$ and ${ }^{15} \mathrm{~N}$ - he measured increased ratios of ${ }^{14} \mathrm{C} /{ }^{15} \mathrm{~N},{ }^{3} \mathrm{H} /{ }^{15} \mathrm{~N}$ and ${ }^{14} \mathrm{C} /{ }^{3} \mathrm{H}$ in nicotine from root to top leaves. Not only did these results fit the distribution pattern of amount of nicotine in these plant parts but demonstrated that newly biosynthesized nicotine was distributed to all plants parts and not just the top leaves. A high percentage of label was found in alkaloids other than nicotine, including nornicotine, anatabine and anabasine. Anatabine had greater incorporation than the other minor alkaloids indicating that biosynthesis of anatabine is more closely related to nicotine biosynthesis than anabasine. This has been proven in subsequent years.

A fundamental but little understood concept of tobacco physiology was the relationship between the amount of essential mineral nutrients and the metabolic changes occurring in the plant as they relate to tobacco as a commodity. It was within this context that T.C. and associates did a series of studies to examine the effects of mineral deficiency on the organic constituents of tobacco. They graphically illustrated changes in alkaloids, carbohydrates, proteins and organic acids with several mineral deficiencies. Deficiencies caused by either B or Ca resulted in cessation of the terminal bud which was physically similar to manual topping. Both manually topped and physiologically topped plants, deficient in $\mathrm{B}$ or $\mathrm{Ca}$, had higher alkaloid accumulation and the alkaloid accumulation was not a simple function of total nitrogen available. Free and protein hydrolyzate amino acids increased in mineral deficient plants, especially the free amino acids. The measured increase in glutamic acid may have been the first to indicator of its role in nicotine biosynthesis.

During early discussions on the health effects of tobacco, the source and amount of radioelements present in tobacco was questioned. Research efforts often had the objective of identifying the source of radiation and finding means for reduction or removal. In a set of well crafted experiments, Dr. Tso and associates examined the source of ${ }^{210} \mathrm{Po}$ and ${ }^{210} \mathrm{~Pb}$ in tobacco leaf. Even with ${ }^{222} \mathrm{Rn}$ supplied to the atmosphere, very little ${ }^{210} \mathrm{Po}$ was found in the tobacco leaf, therefore demonstrating that atmospheric contamination was not the major source of ${ }^{210} \mathrm{Po}$ in tobacco. Because of the cuticle and morphology of the tobacco leaf, the main entrance of these radioelements was from the soil via the 
tobacco root. The conclusions were that cultural practices for the production of tobacco, especially the amount of phosphate fertilizer applied and the cropping system were the main contributors to the accumulation of the radioelements in the leaf.

Dr. Tso was instrumental in developing the procedure known as homogenized leaf curing (HLC). The process includes "curing" tobacco leaf through homogenization, incubation and dehydration. This process allows the reduction or elimination of undesirable constituents or factors of tobacco leaf for commodity use. Most of the nitrogen fractions, including the alkaloids are reduced by HLC. This process led to the removal and utilization of the soluble leaf protein. Proteins contribute little to smoke quality and do contribute to undesirable constituents in the smoke, therefore their removal could provide a higher quality product. The main component of the soluble protein is ribulose-1,5-bisphosphate carboxylase-oxygenase, the enzyme responsible for carbon fixation in tobacco. The biological properties of this soluble protein are excellent and the essential amino acid composition exceeds FAO recommendations as a foodstuff. Based upon these findings much research was spawned on the food and medicinal properties of this protein. This was really the beginning of development of nutraceuticals in tobacco from wild-type or genetically modified tobacco. This early successful protein isolation research was an important impetus to the recent explosion of research activity modifying tobacco to produce value-added products.

His research expertise was rapidly recognized and he was invited to write a chapter on "Physiology of the Tobacco Plant" for Annual Review of Plant Physiology in 1958. In 1972 he published "Physiology and Biochemistry of Tobacco Plants". This was an extremely valuable contribution to the whole tobacco industry as it covered research, utilization and economics. A revised and expanded version was published in 1990. These have been the reference books for over three decades in the debate on tobacco and health. When CORESTA chose to publish a book on tobacco, Dr. Tso wrote the first chapter, "Seed to Smoke".

Obviously, T.C. has been very active in professional organizations. Some tobacco activities in the United States include Chair of two meetings of the Tobacco Science Research Conference, Chair of the TSRC symposium and an author of a symposium presentation. Dr. Tso has received many honors during this career and perhaps most significant among them are the first CORESTA Prize in 1978, appointment as a Charter Member of the Senior Executive Service by President Carter in 1979, and Presidential Rank Award for Meritorious Executive in the Senior Executive Service in the United States by President Reagan in 1984.
Another aspect of Dr. Tso's career has been the strong desire to lead and assist with agricultural development in other countries, especially his homeland China. His first book on this, "Agriculture in China, 1949-2030" covered the period from the founding of the People's Republic of China to their official declaration of promoting agricultural development through science and technology, plus looking forward toward food security in 2030. His emphasis has been and is on the education of the younger generation to improve quality of life for the future.

In his most recent book, "Dare to Dream - Vision of 2050 Agriculture in China", T.C. concurs that the three major concerns to face the world in the next 50 years are food, natural resources and the environment and concludes that agriculture holds the key to resolve all of these. To do such, agriculture must proceed stepwise from the Green Revolution into the Gene Revolution. Importantly, he deduces agricultural education offers key means to link farmers' production plants to consumer demands, as well as to productivity and value enhancing products of academia and research. However, to achieve this, agriculture teaching and research must avoid inbreeding and encourage innovative research-oriented teaching programs. His vision and hope for the next generations rests in the imagination and accomplishments resulting therefrom. This was expressed in the prologue to this book "Many unexpected events have forced us to soar above the trees of tradition. We can glimpse the fresh fields far beyond our imagination. Our eyes must continue to be those of youth, seeing vision, for around us spread new horizons - unlimited."

T.C. has personally encouraged those he has mentored to seek knowledge diligently and to apply that knowledge in our professional and personal lives to the benefit of humanity. His contributions to tobacco science are readily apparent, but his contributions to the lives of others, both in and out of science, are probably more significant. He has high expectations for all, and if we achieve these expectations as he has, this will be a better world for all.

\section{Lowell Bush}

University of Kentucky

Department of Plant and Soil Sciences

College of Agriculture

105 Plant Science Building

Lexington, $K Y$, 40546-0312

USA 